\title{
Editorial: The Incredible Challenge of Digitizing the Human Brain
}

\author{
Luciano Di Mele ${ }^{1 *}$, Carmen Moret-Tatay ${ }^{2,3}$, Mike Murphy $^{4}$, Céline Borg ${ }^{5}$, \\ Raúl Espert-Tortajada ${ }^{6}$ and Camila R. De Oliveira ${ }^{7}$
}

${ }^{1}$ Facoltá di Psicologia, International Telematic University UNINETTUNO, Rome, Italy, ${ }^{2}$ Departamento de Neuropsicobiología, Metodología y Psicología Social, Universidad Católica de Valencia San Vicente Mártir, Valencia, Spain, ${ }^{3}$ Dipartimento di Neuroscienze, Salute Mentale e Organi di Senso (NESMOS), La Sapienza University of Rome, Rome, Italy, ${ }^{4}$ School of Applied Psychology, University College Cork, Cork, Ireland, ${ }^{5}$ CMRR Neuropsychologie Chu de St Etienne, Equipe Vision et Cognition Laboratoire de Psychologie et Neurocognition CNRS 5105 Université UGA, Université Catholique de Lyon, Lyon, France, ${ }^{6}$ Departament de Psicobiologia, Universitat de València, Valencia, Spain, ${ }^{7}$ Mestrado em Psicologia, IMED, Passo Fundo, Brazil

Keywords: social media, media education, multitasking, human cognition, informational nature, digitalization

\section{Editorial on the Research Topic}

\section{The Incredible Challenge of Digitizing the Human Brain}

The world we live in is drastically different from previous decades in terms of digitalization expansion. Logically, one might expect that these changes modify the way we behave, our habits, the way we do tasks, communicate and access information (Moret-Tatay et al., 2018; Wang et al., 2021). And, therefore, the functioning of the brain and even its anatomy. If so, it seems imperative to examine how it affects our cognition. Furthermore, based on the assumption that the brain is plastic and adaptable, some alterations and changes are expected to optimize resources or even compensations by improving other skills (Oliveira et al., 2018; Bubbico et al., 2020; Della Gatta et al., 2021).

It should be noted that these developments take place in the context of a rapidly aging society. Therefore, age-related differences in this technology adoption process are expected, as is the case in previous literature (Charness et al., 2018). Due to the historical moment we live in, we face a once-in-a-lifetime opportunity to address these issues by including theoretical, methodological, and empirical contributions. These might shed light on the following question underlying the current Research Topic: (i) models for human cognition and challenges for the human brain in communication with the digital society; (ii) how media multitasking affects our mental processes.

First, from a theoretical perspective, the first contribution (Byrne) describes a pendulum-like approach to neuron interactions: this involves the rapid firing and restarting of the process and the clusters of neurons in circuits. A digital analogy is proposed through electroencephalogram (EEG) techniques to show frequency changes that are characteristic of different cognitive processes (Golnar-Nik et al., 2019). In this way, each pendulum would represent a process in terms of length, weight, and a damping factor, previously described in terms of quantum search (Chen and Brylinski, 2002). Thus, this approach highlights the field of quantum theory to model cognitive phenomena underlying the information processing by the human brain and its cognitive components such as language, perception, or memory, among others (Jedlicka, 2017). Behavior studies suggest environmental changes, novel or ambiguous situations, which might support an analog new paradigm designed to provide better insights into our subconscious decision-making.

From a behavioral perspective, and according to Ruiz-Ruano García and Puga, our brain is considered a cognitive system with limited processing capacity, and therefore overload can occur. More social aspects are framed as the repercussions for mental health, resulting from increased demand for virtual communication. This may involve other fields such as education and their competencies to search, filter and use high quality online documentation, also described in previous 
literature (Mangen, 2010, 2016). It is clear that this issue is complex and involves many agencies such as communication channels and the education system. Notably, the latter is called on to provide new generations with adequate Information Literacy. On the other hand, this research emphasizes the need to examine the differences in performance between digital and traditional media. In this way, the study carried out by Bernabe-Valero et al. sought to examine differences in response between face-toface and virtual users, supporting previous literature. According to the authors, similar scores are found in both environments supporting the ecological validity of instruments developed in the present such as the G20.

Another view to examine benefits and side effects has been addressed by Popławska et al. This hypothesis and theory article seeks to analyze the conditions of multitasking. According to the authors, when assessing the effectiveness of media multitasking, it is necessary to specify the reference objectives, consequences, and inherent performance. This approach makes it possible to examine multitasking as a strategic behavior undertaken as well as a failure of self-regulation. Digital media has redesigned the way the mind creates innovative social connections. Social communities have multiplied thanks to the web, and thus novel collective resources have contributed to solving problems, sharing skills and creating emerging communities.

Lastly, Beuckels et al. address the role of multitasking in today's society and underlying issues in multitasking. Thus, this piece of research provides a state-of-the-art investigation into multitasking through a systematic review considering the significant increase in papers on this subject in recent years. The primary purpose is to clarify the difference between multitasking and media multitasking and identify the main research trends. A high burden on cognitive resources is described, as suggested by previous literature on this Research Topic, and a review of state of the art is proposed, using a quantitative method, employing a bibliometric and thematic content analysis to identify five major research themes and trends in the general field of media multitasking. The authors identify methodological problems (e.g., differences between survey techniques and

\section{REFERENCES}

Bubbico, G., Chiacchiaretta, P., Parenti, M., di Marco, M., Panara, V., Sepede, G., et al. (2020). Corrigendum: effects of second language learning on the plastic aging brain: functional connectivity, cognitive decline, and reorganization. Front. Neurosci. 14, 108. doi: 10.3389/fnins.2020.00108

Charness, N., Yoon, J. S., Souders, D., Stothart, C., and Yehnert, C. (2018). Predictors of attitudes towards autonomous vehicles: the roles of age, gender, prior knowledge, and personality. Front. Psychol. 9, 2589. doi: 10.3389/fpsyg.2018.02589

Chen, G., and Brylinski, R. K. (2002). Mathematics of Quantum Computation. Cham: Springer International Publishing.

Della Gatta, F., Terribili, C., Fabrizi, E., and Moret-Tatay, C. (2021). Making older adults' cognitive health visible after covid-19 outbreak. Front. Psychol. 12. doi: $10.3389 /$ fpsyg.2021.648208

Floridi, L. (2014). The Fourth Revolution: How the Infosphere is Reshaping Human Reality. Oxford: OUP Oxford.

Golnar-Nik, P., Farashi, S., and Safari, M. S. (2019). The application of EEG power for the prediction and interpretation of consumer decision-making: a neuromarketing study. Physiol. Behav. 207, 90-98. doi: 10.1016/j.physbeh.2019.04.025 cortical measurement of human activity), group differences (highlighting the role of adolescents in this field), theories (e.g., shallowing hypothesis) and types of media.

Digitizing the human brain is still an open challenge, but two aspects are to consider. Firstly, the trap of technological determinism must be avoided (Moret-Tatay and Murphy, 2019). The media identify themselves as the only instigators behind the current historical transformation. Secondly, interdisciplinarity is necessary; the study of mind and digital technologies needs to converge several sciences such as psychology, sociology, neuroscience, education, and philosophy. From current results, it can be concluded that new challenges in the digitization of the human brain will involve changes in theoretical perspective, including the differences between digital and analog approaches, studies on the saturation of cognitive processes and multitasking, breakdowns by groups and media types, as well as the study of ecological validity. In sum, the debate is often framed in dualistic terms, whether the mind or the technology is superior. When combined with the digital realm, the mind forms a continuous dimension; it no longer makes sense to differentiate online or offline. Currently, this unification of personal and social identities, exaggerated by digital media, constitutes a unique form in its informational nature (Floridi, 2014). This unified identity produces emergent behaviors in numerous fields of human life, with outcomes that must be constantly studied and addressed. However, scientific research often focuses on the risks and opportunities of digitalization. Instead, it would be appropriate to broaden the scope of this research to understand this phenomenon in its broader context for all ages (Moret-Tatay et al., 2019). The manuscript selection described in this Research Topic covered many highly complex topics related to this emerging phenomenon.

\section{AUTHOR CONTRIBUTIONS}

All authors listed have made a substantial, direct, and intellectual contribution to the work and approved it for publication.

Jedlicka, P. (2017). Revisiting the quantum brain hypothesis: toward quantum (neuro) biology?. Front. Mol. Neurosci. 10, 366. doi: 10.3389/fnmol.2017.00366

Mangen, A. (2010). Point and click: theoretical and phenomenological reflections on the digitization of early childhood education. Contemporary Issues Early Childhood 11, 415-431. doi: 10.2304/ciec.2010.11.4.415

Mangen, A. (2016). The digitization of literary reading: contributions from empirical research. Orbis litterarum 71, 240-262. doi: 10.1111/oli.12095

Moret-Tatay, C., Beneyto-Arrojo, M. J., Gutierrez, E., Boot, W. R., and Charness, N. (2019). A spanish adaptation of the computer and mobile device proficiency questionnaires (CPQ and MDPQ) for older adults. Front. Psychol. 10, 1165. doi: 10.3389/fpsyg.2019.01165

Moret-Tatay, C., Gamermann, D., Murphy, M., and Kuzmičov,á, A. (2018). Just google it: an approach on word frequencies based on online search result. $J$. Gen. Psychol. 145, 170-182. doi: 10.1080/00221309.2018.1459451

Moret-Tatay, C., and Murphy, M. (2019). Aging in the digital era. Front. Psychol. 10, 1815. doi: 10.3389/fpsyg.2019.01815

Oliveira, C. R. D., Lopes Filho, B. J. P., Esteves, C. S., Rossi, T., Nunes, D. D. S., de Lima, M. M. B. M., et al. (2018). Neuropsychological assessment of older adults with virtual reality: association of age, schooling, and general cognitive status. Front. Psychol. 9, 1085. doi: 10.3389/fpsyg.2018.0 1085 
Wang, Y., Dai, Y., Li, H., and Song, L. (2021). Social media and attitude change: information booming promote or resist persuasion?. Front. Psychol. 12, 2433. doi: $10.3389 /$ fpsyg.2021.596071

Conflict of Interest: The authors declare that the research was conducted in the absence of any commercial or financial relationships that could be construed as a potential conflict of interest.

Publisher's Note: All claims expressed in this article are solely those of the authors and do not necessarily represent those of their affiliated organizations, or those of the publisher, the editors and the reviewers.
Any product that may be evaluated in this article, or claim that may be made by its manufacturer, is not guaranteed or endorsed by the publisher.

Copyright (๑ 2022 Di Mele, Moret-Tatay, Murphy, Borg, Espert-Tortajada and De Oliveira. This is an open-access article distributed under the terms of the Creative Commons Attribution License (CC BY). The use, distribution or reproduction in other forums is permitted, provided the original author(s) and the copyright owner(s) are credited and that the original publication in this journal is cited, in accordance with accepted academic practice. No use, distribution or reproduction is permitted which does not comply with these terms. 